\title{
Evaluation of the Effect of Alogliptin on Tissue Characteristics of the Carotid Wall: Subanalysis of the SPEAD-A Trial
}

\author{
Yoko Irie $\cdot$ Naoto Katakami (D) Tomoya Mita · Mitsuyoshi Takahara • \\ Taka-aki Matsuoka • Masahiko Gosho · Hirotaka Watada · Iichiro Shimomura • \\ Study of Preventive Effects of Alogliptin on Diabetic Atherosclerosis (SPEAD-A) Collaborators
}

Received: November 14, 2017 / Published online: January 12, 2018

(c) The Author(s) 2018. This article is an open access publication

\section{ABSTRACT}

Introduction: Ultrasonic tissue characterization of the carotid wall using gray-scale median (GSM) reflects its composition and low-GSM plaque is considered to be unstable. The present study evaluated the effect of alogliptin, a dipeptidyl peptidase- 4 inhibitor, on the longitudinal change in GSM, an index of the tissue characteristics of the carotid wall, in patients with type 2 diabetes (T2DM).

Enhanced content To view enhanced content for this article go to http://www.medengine.com/Redeem/ 402DF06027669E70.

Y. Irie

Department of Endocrinology and Diabetes, Osaka Police Hospital, 10-31 Kitayamacho, Tennoji-ku, Osaka 543-0035, Japan

N. Katakami $(\bowtie) \cdot$ M. Takahara · T. Matsuoka . I. Shimomura

Department of Metabolic Medicine, Osaka

University Graduate School of Medicine, 2-2,

Yamadaoka, Suita, Osaka 565-0871, Japan

e-mail: katakami@endmet.med.osaka-u.ac.jp

N. Katakami

Department of Metabolism and Atherosclerosis,

Osaka University Graduate School of Medicine, 2-2,

Yamadaoka, Suita, Osaka 565-0871, Japan

T. Mita $\cdot$ H. Watada

Department of Metabolism and Endocrinology, Juntendo University Graduate School of Medicine, Hongo 2-1-1, Bunkyo-ku, Tokyo 113-8421, Japan
Methods: This is a post hoc subanalysis using data obtained from the SPEAD-A trial, a randomized controlled trial that demonstrated the beneficial effect of alogliptin treatment on the progression of carotid intima-media thickness in patients with T2DM with no past history of apparent cardiovascular disease. A total of 322 subjects (161 in the alogliptin treatment group and 161 in the conventional treatment group) were enrolled. The primary outcome was the change from baseline in mean GSM-CCA (common carotid artery) during the 104-week observation period.

Results: Both alogliptin treatment and conventional treatment significantly increased the mean GSM-CCA (from $60.7 \pm 12.3$ to

T. Mita $\cdot$ H. Watada

Center for Molecular Diabetology, Juntendo

University Graduate School of Medicine, Hongo 2-1-

1, Bunkyo-ku, Tokyo 113-8421, Japan

M. Takahara

Department of Diabetes Care Medicine, Osaka

University Graduate School of Medicine, 2-2,

Yamadaoka, Suita, Osaka 565-0871, Japan

M. Gosho

Department of Clinical Trial and Clinical Epidemiology, Faculty of Medicine, University of Tsukuba, 1-1-1, Tennodai, Tsukuba, Ibaraki 3058575, Japan

H. Watada

Center for Therapeutic Innovations in Diabetes, Juntendo University Graduate School of Medicine, Hongo 2-1-1, Bunkyo-ku, Tokyo 113-8421, Japan 
$65.9 \pm 10.1, p<0.001$ and $58.8 \pm 14.4-65.2 \pm$ $12.2, p<0.001$, respectively) and there was no significant difference in changes in mean GSMCCA between the treatment groups $(p=0.95)$. Additionally, there were no differences in the changes in the left and right GSM-CCA between the groups.

Conclusions: A post hoc subanalysis revealed an improvement of tissue characteristics in the carotid arterial wall in both the alogliptin treatment group and the conventional treatment group during the 104-week treatment period and that there was no significant difference between the treatment groups.

Clinical Trial Registration: UMIN000019951.

Keywords: DPP-4 inhibitor; Carotid artery; Diabetes mellitus; Tissue characteristics; Carotid ultrasound

\section{INTRODUCTION}

Cardiovascular disease (CVD) is the main cause of death and impairment of quality of life in patients with diabetes mellitus (DM). Therefore, prevention and management of cardiovascular risk are critical in these patients.

It is well known that disruption of vulnerable atherosclerotic plaque plays a crucial role in the pathogenesis of CVD events and that plaque disruption is dependent on the tissue characteristics of the plaque lesion: the lipid content, the presence of neovascular vessels, inflammatory cells in the atheroma, and the thickness of the fibrous cap [1-4]. Since diabetes is related to increased vulnerability to plaque disruption and higher incidence of clinical CVD [5], early identification of vulnerable plaque and subsequent prompt intervention are important to reducing the incidence of CVD events in the management of diabetes.

A recent study indicates that noninvasive ultrasonic tissue characterization of carotid plaque using gray-scale median (GSM) reflects plaque composition and that low-GSM plaque, which consists mainly of neovascular vessels, has a high lipid content, and is characterized by inflammatory infiltration, is considered to be particularly unstable [6]. Furthermore, the GSM value in the carotid wall can serve as a predictor of future CVD events in subjects with and without diabetes, even after adjustment for traditional risk factors [7-9].

Dipeptidyl peptidase-4 (DPP-4) inhibitors, which inhibit the degradation of active incretins including glucagon-like polypeptide-1 (GLP-1) by DPP-4, increase the concentration of active incretins and thereby enhance their glucoregulatory effects. In addition to the antidiabetic properties mentioned above, these incretin mimetics potentially have antiatherosclerotic properties [10]. First, GLP-1 agonists can significantly decrease body weight and DPP-4 inhibitors are considered weight neutral, both of which are advantageous properties given that weight gain is often seen with other antidiabetic agents $[11,12]$. Second, GLP1 acts directly on endothelial cells, vascular smooth muscle cells, monocytes, macrophages, and lymphocytes, and GLP-1 and GLP-1 receptor agonists have been shown to inhibit foam cell formation and atherosclerosis by suppressing inflammation and oxidative stress [13-20]. In addition, several in vitro and in vivo experiments have shown that DPP-4 inhibitors can also inhibit foam cell formation and atherosclerosis in both a GLP-1-dependent and GLP-1-independent manner [21-26]. Vittone et al. demonstrated that treatment of apolipoprotein E-deficient mice with a DPP-4 inhibitor reduced plaque inflammation and increased plaque stability, potentially by GLP-1mediated inhibition of chemokine-induced monocyte migration and macrophage MMP-9 release [26]. Such anti-inflammatory and antiatherosclerotic effects of DPP-4 inhibitors have also been confirmed by studies conducted in clinical settings $[25,27,28]$. Interestingly, Balestrieri et al. evaluated the effect of incretinbased therapies in carotid plaques of asymptomatic patients undergoing carotid endarterectomy. They found that in comparison with non-diabetic plaques, plaques in patients with DM had increased inflammation and oxidative stress along with reduced collagen content. Compared with non-incretintreated plaques, incretin therapy-treated plaques presented with higher collagen content, 
and less inflammation and oxidative stress, indicating a more stable plaque phenotype [25].

We recently reported that treatment with alogliptin, a DPP-4 inhibitor, attenuated the progression of carotid intima-media thickness (IMT) compared with conventional treatment in patients with type 2 diabetes mellitus (T2DM) [29]. The aim of the Study of Preventive Effects of Alogliptin on Diabetic Atherosclerosis (SPEAD-A), a prospective, randomized, openlabel, multicenter, blinded end point study, was to evaluate the efficacy of alogliptin in preventing the progression of atherosclerosis in T2DM patients with no past history of apparent CVD. The primary outcome was the change in IMT during the 2-year intervention period. This trial demonstrated that alogliptin potently inhibited increase in carotid IMT compared with conventional treatment and that there was substantial regression of IMT at the end of the alogliptin treatment period [29]. Although the exact mechanism is not well understood at present, it has been hypothesized that the regression of carotid IMT induced by alogliptin treatment could be related to stabilization of atheromatous lipid-rich lesions via suppression of inflammation and foam cell formation. However, little is known about the effect of alogliptin on the tissue characteristics of atherosclerotic lesions in the carotid wall.

To address this point, we evaluated the effect of alogliptin on the longitudinal change in the GSM value, an index of the ultrasonic tissue characteristics of the carotid wall, in patients with T2DM, using data obtained from the SPEAD-A trial.

\section{METHODS}

\section{Subjects}

We performed a post hoc analysis based on data obtained from the SPEAD-A trial [29]. Although the study design, inclusion and exclusion criteria, study schedule, and measurements of the SPEAD-A trial have been described in detail previously [29], the outline is as follows. Participants eligible for the study were those who had T2DM, were aged 30 years or older at the time of enrollment, had been treated with agents other than DPP-4 inhibitors administered for 3 months or longer, and whose HbA1c was below $9.4 \%$. Exclusion criteria were (1) type 1 diabetes mellitus or secondary diabetes, (2) severe infections before or after surgery or severe trauma, (3) myocardial infarction, angina pectoris, cerebral stroke, or cerebral infarction, (4) moderate or severe renal dysfunction (serum creatinine: men, $\geq 1.4 \mathrm{mg} / \mathrm{dL}$; women, $\geq 1.2 \mathrm{mg} / \mathrm{dL}$ ), (5) severe liver dysfunction (aspartate aminotransferase $\geq 100 \mathrm{IU} / \mathrm{L}$ ), (6) moderate or severe heart failure (New York Heart Association stage III or higher), (7) under treatment with an incretin preparation, such as other DPP-4 inhibitors, at the start of the study, (8) receiving insulin treatment, (9) receiving therapeutic drugs not concomitantly administrable with incretin preparations with regard to the National Health Insurance program, such as DPP-4 inhibitors, at the start of the study, (10) pregnant, lactating, or possibly pregnant women or those planning to become pregnant, (11) medical history of hypersensitivity to investigational drugs, or (12) judged as ineligible by clinical investigators.

Japanese T2DM patients with no past history of apparent CVD who periodically attended the Outpatient Diabetes Clinics at 11 centers across Japan were asked to participate in this study and all patients who agreed to participate were enrolled in the study. Originally, a total of 341 patients were enrolled and randomly allocated into either the alogliptin group $(n=172)$ or the conventional treatment group (using drugs other than the DPP-4 inhibitor) $(n=169)$. After excluding 19 patients from analyses (they withdrew from the study and/or objected to the inclusion of their data in any analysis), 161 subjects in the alogliptin treatment group and 161 in the conventional treatment group were included in the full analysis set.

The protocol was approved by the institutional review board of each participating institution in compliance with the Declaration of Helsinki and current legal regulations in Japan. Written informed consent was obtained from all the participants after full explanation of the study. This study has been registered on the University Hospital Medical Information 
Network Clinical Trials Registry, which is a nonprofit organization in Japan and meets the requirements of the International Committee of Medical Journal Editors (UMIN000007396, UMIN000019951).

\section{Ultrasound Examination}

B-mode ultrasonography of the carotid artery was performed using an ultrasound machine with a high-frequency $(>7.5 \mathrm{MHz})$ linear transducer. In accordance with the guidelines of the Japan Society of Ultrasonics [30], scanning of the extracranial common carotid artery (CCA), the carotid bulb, and the internal carotid artery in the neck was performed bilaterally in three different longitudinal projections as well as transverse projections. The IMT was measured as the distance between two parallel echogenic lines corresponding to the blood-intima and media-adventitia interface on the posterior wall of the artery. The measurements of mean IMT of the CCA (mean IMT-CCA) were performed using automated digital edge-detection software (IntimaScope; MEDIA CROSS, Tokyo, Japan) [31]. The software system averaged 200 points of IMT values in the segment $2 \mathrm{~cm}$ proximal to the dilation of the carotid bulb. The measurements of maximum IMT of the CCA (max IMT-CCA) were performed at the site of the thickest point in the CCA. The method for determining IMT has been described in detail in previous reports [29].

The echogenicity of the arterial wall was evaluated on the basis of the GSM method in a gray-scale range of 0-255 ( 0 as the darkest and 255 as the brightest tone). Adobe Photoshop software (Adobe Systems, version 7.0, San Jose, CA, USA) was used for image standardization and calculation of gray-scale values. In accordance with the previous report, the standardization of the B-mode image was performed using a curve option, so that the GSM for the blood ranged from 0 to 5 , and for the adventitia from 185 to 195 [32]. Then, the mean IMT-CCA area (the segment $2 \mathrm{~cm}$ proximal to the dilation of the carotid bulb) was delineated with a freehand tool, and the GSM of the selected area was read from the entire delineated area. Similarly, if there was an atherosclerotic plaque lesion (i.e., focal elevated lesion with max IMT-CCA $>1.0 \mathrm{~mm}$ ), the GSM of the plaque lesion was also measured using the same method. In the event multiple plaque lesions were found in one individual, the plaque with the greatest thickness was subject to GSM measurement and the GSM value was used as the subject's representative value. To avoid inter-reader variability, all scans were electronically stored and read in random order by a single reader (Y.I.) who was unaware of the clinical characteristics and the treatment group of the subjects.

\section{Outcome Measures}

The primary outcome of this study was the change of GSM value in the mean IMT-CCA area (in particular, the arithmetic average of the right and the left GSM values in one individual) during the 104-week observation period. In cases where atherosclerotic plaque lesions were found, the changes of the GSM value in the plaques were also evaluated. The definitions of the GSM measures used in this study are as follows:

- Right GSM-CCA: the GSM value of the intima-media complex measured in the mean IMT-CCA area (the segment $2 \mathrm{~cm}$ proximal to the dilation of the carotid bulb) of the right common carotid artery.

- Left GSM-CCA: the GSM value of the intimamedia complex measured in the mean IMTCCA area (the segment $2 \mathrm{~cm}$ proximal to the dilation of the carotid bulb) of the left common carotid artery.

- Mean GSM-CCA: the arithmetic average of the right and left GSM-CCA values. (If either the right or left GSM-CCA value was not obtained, the value of the other side was used as the mean GSM-CCA value.)

- Right GSM-plaque: the GSM value of the plaque lesion ( $\max$ IMT-CCA $>1.0 \mathrm{~mm}$ ) with the greatest thickness measured in the right common carotid artery.

- Left GSM-plaque: the GSM value of the plaque lesion ( $\max$ IMT-CCA $>1.0 \mathrm{~mm}$ ) with the greatest thickness measured in the left common carotid artery. 


\section{Statistical Analyses}

All values are reported as mean $\pm S D$, median (range), or actual number of subjects with the percentage in parentheses.

The primary end point was the change in GSM of the carotid wall from baseline to 104 weeks. The primary analysis was performed using the mixed-effects model for repeated measures (MMRM) including treatment group, time (week), baseline GSM, and interaction between treatment group and time with an unstructured covariance structure to model within-subject variability. Baseline and followup group comparisons were assessed with the Student $t$ test or Wilcoxon rank sum test for continuous variables and Fisher's exact test for categorical variables.

To evaluate the associations between change in the mean GSM-CCA and other clinical parameters such as age, gender, body mass index (BMI), HbA1c, serum lipid levels (e.g., TC, HDL-C, TG), blood pressure, smoking status, and administration of antidiabetic, antihypertensive, antihyperlipidemic, and antiplatelet drugs, and mean IMT-CCA, regression analyses including treatment group as a covariate were performed.

All statistical tests were two-sided with a 5\% significance level. Analyses were performed using SAS 9.4 software (SAS Institute Inc., Cary, $\mathrm{NC)}$.

\section{Compliance with Ethics Guidelines}

This article is based on previously conducted studies and does not involve any new studies of human or animal subjects performed by any of the authors.

\section{RESULTS}

\section{Baseline Characteristics of Study Subjects}

The baseline demographic and clinical characteristics of the 322 study participants have been previously reported (161 subjects in the alogliptin group and 161 in the conventional treatment group) [29]. At baseline, there were no significant differences between the alogliptin group and the conventional treatment group in terms of the clinical parameters: age was $64.4 \pm 9.8$ and $64.8 \pm 9.1(p=0.72)$, the percentage of males was $63 \%$ and $61 \%$ $(p=0.82), \quad$ HbA1c $\quad$ was $7.3 \pm 0.8 \%$ and $7.2 \pm 0.8 \%(p=0.54)$, BMI was $24.6 \pm 4.3$ and $24.9 \pm 3.7(p=0.49)$, the prevalence of hypertension was $56 \%$ and $57 \%(p=1.00)$, the prevalence of dyslipidemia was $53 \%$ and $58 \%$ $(p=0.43)$, and the percentage of statin use was $38 \%$ and $46 \%(p=0.18)$, respectively.

Alogliptin treatment had a more potent glucose-lowering effect than the conventional treatment $\quad(-0.3 \pm 0.7 \%$ vs. $-0.1 \pm 0.8 \%$, $p=0.004)$ without an increase in hypoglycemia [29]. However, the increase in BMI at 104 weeks was greater in the alogliptin group than in the conventional treatment group $(0.3 \pm 1.9$ vs. $\left.-0.3 \pm 1.7 \mathrm{~kg} / \mathrm{m}^{2}, p=0.003\right)$. The increase in serum VCAM-1 levels at 104 weeks was greater in the alogliptin group than in the conventional treatment group $(20$ vs. $-8 \mathrm{ng} / \mathrm{mL}$, $p=0.030)$. Regarding other markers of inflammation and endothelial injury such as hs-CRP, interleukin-6, and ICAM-1, however, there were no significant differences between the treatment groups in differences in parameters from baseline to 104 weeks (data not shown).

\section{Effect of Alogliptin on the Carotid Wall}

According to the results of the SPEAD-A trial, reductions in the mean common and the right and left maximum IMT of the carotid arteries at 104 weeks were significantly greater after alogliptin treatment than after conventional treatment $[-0.026$ (SE 0.009) vs. $0.005 \mathrm{~mm}$ (SE $0.009), \quad p=0.022 ; \quad-0.045$ (SE 0.018) vs. $0.011 \mathrm{~mm}$ (SE 0.017), $p=0.025$, and -0.079 (SE 0.018 ) vs. $-0.015 \mathrm{~mm}$ (SE 0.018), $p=0.013$, respectively] [29].

At baseline, mean GSM-CCA and left GSMCCA values were measurable in all the study subjects but right GSM-CCA values were not measurable in two subjects in the alogliptin treatment group. Atherosclerotic plaque lesions were observed in the right CCA in 61 subjects 
Table 1 Effect of alogliptin on the GSM of the carotid wall

\begin{tabular}{|c|c|c|c|c|c|c|}
\hline & $n$ & $\begin{array}{l}\text { Alogliptin } \\
\text { treatment group }\end{array}$ & $n$ & $\begin{array}{l}\text { Conventional } \\
\text { treatment group }\end{array}$ & $\begin{array}{l}\text { Adj. mean } \\
\text { difference }(95 \% \mathrm{CI})\end{array}$ & $\begin{array}{l}p \text { value } \\
\text { between groups }\end{array}$ \\
\hline \multicolumn{7}{|c|}{ Mean GSM-CCA } \\
\hline Baseline & 161 & $60.7 \pm 12.3$ & 161 & $58.8 \pm 14.0$ & & \\
\hline 52 weeks & 153 & $65.7 \pm 11.6^{* *}$ & 157 & $64.2 \pm 11.2^{* *}$ & $0.73(-1.62,3.07)$ & 0.54 \\
\hline 104 weeks & 150 & $65.9 \pm 10.1^{* *}$ & 154 & $65.2 \pm 12.2^{* *}$ & $0.07(-2.23,2.38)$ & 0.95 \\
\hline \multicolumn{7}{|c|}{ Right GSM-CCA } \\
\hline Baseline & 159 & $61.2 \pm 13.4$ & 161 & $58.7 \pm 15.3$ & & \\
\hline 52 weeks & 152 & $65.5 \pm 12.3^{* *}$ & 156 & $63.9 \pm 11.9^{* *}$ & $0.89(-1.69,3.46)$ & 0.50 \\
\hline 104 weeks & 150 & $65.6 \pm 10.9^{* *}$ & 154 & $64.7 \pm 13.8^{* *}$ & $0.24(-2.36,2.83)$ & 0.86 \\
\hline \multicolumn{7}{|c|}{ Left GSM-CCA } \\
\hline Baseline & 161 & $60.5 \pm 14.1$ & 161 & $58.9 \pm 15.5$ & & \\
\hline 52 weeks & 152 & $65.9 \pm 14.0^{* *}$ & 157 & $64.7 \pm 13.3^{* *}$ & $0.65(-2.21,3.51)$ & 0.66 \\
\hline 104 weeks & 150 & $66.2 \pm 12.9^{* *}$ & 154 & $65.7 \pm 13.9^{* *}$ & $0.04(-2.71,2.80)$ & 0.98 \\
\hline \multicolumn{7}{|c|}{ Right GSM-plaque } \\
\hline Baseline & 25 & $66.1 \pm 14.9$ & 36 & $60.9 \pm 13.7$ & & \\
\hline 52 weeks & 23 & $68.3 \pm 13.6$ & 35 & $61.4 \pm 13.2$ & $5.81(-1.05,12.67)$ & 0.10 \\
\hline 104 weeks & 22 & $68.1 \pm 9.7$ & 34 & $63.1 \pm 11.8$ & $4.54(-1.50,10.58)$ & 0.14 \\
\hline \multicolumn{7}{|c|}{ Left GSM-plaque } \\
\hline Baseline & 38 & $66.3 \pm 19.0$ & 38 & $59.7 \pm 15.1$ & & \\
\hline 52 weeks & 35 & $67.1 \pm 14.4$ & 37 & $67.5 \pm 11.6^{* *}$ & $-2.68(-8.65,3.29)$ & 0.37 \\
\hline 104 weeks & 33 & $70.4 \pm 19.4$ & 37 & $67.0 \pm 13.4^{*}$ & $1.03(-6.31,8.36)$ & 0.78 \\
\hline
\end{tabular}

Data are mean $\pm S D$ unless otherwise stated. Comparisons of GSMs during treatment with those at baseline were performed with one-sample $t$ test based on a mixed-effects model for repeated measures. Differences in $\Delta$ change in GSM from baseline at 52 and 104 weeks between groups were analyzed with a mixed-effects model for repeated measures. Treatment group, week, interactions between treatment group and week, and baseline GSM were included as fixed effects ${ }^{*} p<0.05 ;{ }^{* *} p<0.01$

(25 subjects in the alogliptin group and 36 in the conventional treatment group) and in the left CCA in 76 subjects (38 subjects in the alogliptin group and 38 in the conventional treatment group). The GSM values of these plaques were measured. There were no significant differences in all the GSM measures (i.e., mean GSM-CCA, right GSM-CCA, left GSM-CCA, right GSM-plaque, and left GSM-plaque) between the two treatment groups at baseline (Table 1).
Both alogliptin treatment and conventional treatment significantly increased the mean GSM-CCA (from $60.7 \pm 12.3$ to $65.9 \pm 10.1$, $p<0.001$ in the alogliptin treatment group and $58.8 \pm 14.4$ to $65.2 \pm 12.2, p<0.001$ in the conventional treatment group), right GSM-CCA (from $61.2 \pm 13.4$ to $65.6 \pm 10.9, p<0.001$ in the alogliptin treatment group and from $58.7 \pm 15.3$ to $64.7 \pm 13.8, p<0.001$ in the conventional treatment group), and left GSMCCA (from $60.5 \pm 14.1$ to $66.2 \pm 12.9, p<0.001$ 
in the alogliptin treatment group and from $58.9 \pm 15.5$ to $65.7 \pm 13.9, p<0.001$ in the conventional treatment group) during the 104-week observation period. There was also a significant increase in left GSM-plaque in the conventional treatment group (from $59.7 \pm 15.1$ to $67.0 \pm 13.4, p=0.01$ ).

The magnitude of the change in GSM values during the treatment period between the two treatment groups was compared using the MMRM (Table 1). These analyses demonstrated that both alogliptin treatment and conventional treatment significantly increased all the GSM measures except for right GSM-plaque (in the conventional treatment group). However, there was no significant difference in the change in GSM measures from baseline at 52 and 104 weeks between the two groups. Furthermore, similar findings were shown even after adjustment for possible confounding factors such as age, gender, BMI, HbA1c, serum lipid levels (e.g., TC, HDL-C, TG), blood pressure, smoking status, and administration of antidiabetic, antihypertensive, antihyperlipidemic, and antiplatelet drugs (data not shown). Finally, we divided the subjects into the tertiles according to the level of GSM at baseline, and investigated the effect of alogliptin on low-GSM (echolucent) and high-GSM (echorich) plaque separately (Table 2 ). The change in right-GSMplaque in the first tertile (lowest GSM group) was significantly greater in the alogliptin group than in the conventional treatment group [26.4 (SE 4.6) vs. 10.9 (SE 2.9), $p=0.01$ ]. However, there was no significant difference in the changes in right GSM-plaque in the second and third tertiles, and left GSM-plaque in any tertiles, between the two treatment groups.

Regression analyses revealed that gender and age at baseline (regression coefficient $\pm \mathrm{SE}$; $3.93 \pm 1.55, \quad p=0.012$ and $0.17 \pm 0.08$, $p=0.04$, respectively) were positively related to changes in mean GSM-CCA and diastolic blood pressure at baseline $(-0.17 \pm 0.07, p=0.01)$ was negatively related to changes in mean GSMCCA. However, there was no statistically significant association between the other clinical parameters including baseline mean IMT-CCA and mean GSM-CCA.
We also evaluated the relationship between the changes in GSM during 104 weeks and those in IMT/plaque thickness in the same site. The changes in mean GSM-CCA, right GSM-CCA, and left GSM-plaque were significantly associated with those in IMT/plaque thickness in the same site $(r=-0.14, p=0.02 ; \quad r=-0.13$, $p=0.02 ; \quad r=-0.28, \quad p=0.02$, respectively), while the changes in left GSM-CCA and left GSM-plaque were not.

\section{DISCUSSION}

We previously demonstrated that alogliptin, a DPP-4 inhibitor, more potently inhibited the progression of carotid IMT than conventional treatment in patients with T2DM [29]. However, few studies have evaluated the effect of DPP-4 inhibitors on the tissue characteristics of the arterial wall.

The present study, a post hoc subanalysis using data obtained from a randomized controlled trial that evaluated the efficacy of alogliptin treatment on the progression of carotid IMT in patients with T2DM, showed that alogliptin treatment significantly increased the GSM value, an index of ultrasonic tissue characteristics, of the carotid arterial wall over a 104-week observation period. However, interestingly, conventional treatment also increased GSM of the carotid arterial wall during this 104-week period and there were no significant differences in the changes of GSM measures between the two treatment groups.

Although the precise mechanism of the formation of vulnerable plaque with a lipid-rich core is unclear, it has been hypothesized that hypercholesterolemia, oxidative stress, inflammation, and insulin resistance are associated with its formation [33]. Clinical studies have also shown that the composition of carotid plaque is related to serum lipid profiles, BMI, and inflammation markers. Our previous study revealed that the presence of echolucent lowGSM plaques in carotid arteries was related to serum lipid profiles and BMI [34]. Interestingly, in the present study, total cholesterol levels at the 52-, 78-, and 104-week observation points were significantly decreased from the baseline 
Table 2 Effect of alogliptin on the carotid plaque according to the level of baseline GSM

\begin{tabular}{|c|c|c|c|c|c|c|}
\hline & $n$ & $\begin{array}{l}\text { Mean change (SE) } \\
\text { of alogliptin } \\
\text { treatment group }\end{array}$ & $n$ & $\begin{array}{l}\text { Mean change (SE) } \\
\text { of conventional } \\
\text { treatment group }\end{array}$ & $\begin{array}{l}\text { Adj. mean difference } \\
(95 \% \mathrm{CI})\end{array}$ & $\begin{array}{l}p \text { value between } \\
\text { groups }\end{array}$ \\
\hline \multicolumn{7}{|c|}{ Right GSM-plaque } \\
\hline \multicolumn{7}{|l|}{ First tertile } \\
\hline 52 weeks & 5 & $20.6(5.2)^{* *}$ & 14 & $6.6(3.1)^{*}$ & $14.1(1.3,26.8)$ & 0.03 \\
\hline 104 weeks & & $26.4(4.6)^{* *}$ & & $10.9(2.9)^{* *}$ & $15.6(3.9,27.2)$ & 0.01 \\
\hline \multicolumn{7}{|c|}{ Second tertile } \\
\hline 52 weeks & 10 & $0.1(4.2)$ & 11 & $-0.1(4.0)$ & $0.1(-12.1,12.3)$ & 0.99 \\
\hline 104 weeks & & $0.3(3.1)$ & & $-3.1(3.0)$ & $3.4(-5.6,12.4)$ & 0.44 \\
\hline \multicolumn{7}{|l|}{ Third tertile } \\
\hline 52 weeks & 8 & $-5.2(4.7)$ & 10 & $-8.8(4.2)$ & $3.6(-9.8,17.0)$ & 0.57 \\
\hline 104 weeks & & $-9.2(4.2)^{*}$ & & $-6.6(3.5)$ & $-2.6(-14.4,9.2)$ & 0.65 \\
\hline \multicolumn{7}{|c|}{ Left GSM-plaque } \\
\hline \multicolumn{7}{|l|}{ First tertile } \\
\hline 52 weeks & 11 & $18.0(4.0)^{* *}$ & 14 & $21.2(3.6)^{* *}$ & $-3.2(-14.5,8.0)$ & 0.56 \\
\hline 104 weeks & & $17.1(4.6)^{* *}$ & & $19.1(4.1)^{* *}$ & $-2.0(-14.7,10.8)$ & 0.75 \\
\hline \multicolumn{7}{|c|}{ Second tertile } \\
\hline 52 weeks & 11 & $-0.4(3.5)$ & 12 & $1.5(3.3)$ & $-1.9(-11.9,8.1)$ & 0.69 \\
\hline 104 weeks & & $0.9(4.0)$ & & $3.5(3.7)$ & $-2.7(-14.0,8.7)$ & 0.63 \\
\hline \multicolumn{7}{|l|}{ Third tertile } \\
\hline 52 weeks & 13 & $-10.6(4.1)^{*}$ & 11 & $-6.4(4.5)$ & $-4.2(-18.0,9.6)$ & 0.53 \\
\hline 104 weeks & & $-2.1(5.3)$ & & $-7.6(5.7)$ & $5.5(-11.5,22.4)$ & 0.51 \\
\hline
\end{tabular}

Data are mean (SE) unless otherwise stated. Comparisons of GSMs during treatment with those at baseline were performed with one-sample $t$ test based on a mixed-effects model for repeated measures. Differences in $\Delta$ change in GSM from baseline at 52 and 104 weeks between groups were analyzed with a mixed-effects model for repeated measures. Treatment group, week, interactions between treatment group and week, and baseline GSM were included as fixed effects ${ }^{*} p<0.05 ;{ }^{* *} p<0.01$

in the conventional treatment group [29]. Similarly, total cholesterol levels at 52 and 78 weeks were significantly decreased from the baseline in the alogliptin treatment group [29]. Therefore, in both treatment groups, reduction in serum total cholesterol levels during the treatment period may have led to an increase in GSM of the carotid arterial wall.

This post hoc subanalysis of the SPEAD-A trial showed that the tissue characteristics of the arterial wall were improved in both treatment groups, although the original study had clearly demonstrated that alogliptin treatment more potently inhibited the progression of carotid IMT than conventional treatment in patients with T2DM [29]. In addition, there was a weak but statistical significant association between changes in GSM and those in IMT or plaque thickness, suggesting that the improvement of tissue characteristics of the carotid wall 
contributed to the regression of the carotid wall thickness. However, the determinants of the tissue characteristics of the carotid wall and those of the carotid IMT are not the same. Although regression of carotid IMT is supposed to be subsequent to pathological changes such as reduction of cholesterol accumulation in the local site, the risk factors for the progression of carotid IMT are reported to include several parameters including average HbA1c levels during the observation period [35]. In our study, although a reduction in serum total cholesterol levels, one of the most important determinants for tissue characteristics of arterial wall, was observed in both treatment groups, reduction in HbA1c was observed only in the alogliptin treatment group [29]. For regression of carotid IMT, therefore, improvement in hyperglycemia as well as a reduction in serum total cholesterol levels may be necessary in patients with DM. Direct anti-atherosclerotic effects of alogliptin on vascular cells is another possible explanation for its beneficial effect on carotid IMT.

There is also a possibility that the beneficial effect of alogliptin on the tissue characteristics of the carotid wall was masked by some confounding factors. For example, the improvements in the determinants of plaque echogenicity such as serum lipid profiles and BMI were relatively greater in the conventional treatment group as compared to the alogliptin treatment group in this study, although between-group differences did not reach statistical significance. Furthermore, the administration rate of statins during the treatment period was also relatively higher in the conventional treatment group than in the alogliptin treatment group: the percentage of statin users was $38 \%$ in the alogliptin treatment group and $46 \%$ in the conventional treatment group $(p=0.18)$ at baseline, and $39 \%$ and $50 \%(p=0.06)$ at 104 weeks. Since statins have a potent antiatherogenic effect and have been reported to decrease IMT and improve the tissue characteristics of plaques in the carotid artery [36-38], such an uncontrolled imbalance in the administration of statins might have masked the potential beneficial effect of alogliptin.
There is also a possibility that the beneficial effect of alogliptin on the carotid wall was dependent on its characteristics at baseline. Therefore, we evaluated effect of alogliptin on low-GSM (echolucent) and high-GSM (echorich) plaque separately. Interestingly, change in the right GSM-plaque in the first tertile (lowest GSM group) was significantly greater in the alogliptin group than in the conventional treatment group but there was no significant treatment-group difference in the changes of right GSM-plaque in the second and third tertiles. Although these findings suggested a possible beneficial effect of alogliptin on low-GSM (echolucent) plaque in the right carotid, this was not the case with the left carotid. This discrepancy could be due to lack of statistical power rather than bilateral difference, since the number of the subjects in each subgroup was very small. To evaluate whether the beneficial effect of alogliptin on the carotid wall was dependent on its characteristics at baseline, another study with larger scale is necessary.

Several limitations of our study should be discussed. First, the present study is a post hoc subanalysis using data obtained from the SPEAD-A trial. Second, the ultrasound settings for each image were not always standardized. However, the blood was used as the reference for black and the adventitia as the reference for white, and gain settings for measurements within an individual were similar throughout the study. Therefore, the impact of gain of ultrasound beam on the GSM value would be quite small, if any. Third, although administration of antidiabetic, antihyperlipidemic, and antihypertensive drugs may affect the plaque components, the baseline medical prescriptions were not matched completely. In addition, it was not possible to adjust for the effect of changes in therapeutic regimen during the observation period. These points should be confirmed in further studies. Fourth, relatively low-risk patients were enrolled in the SPEAD-A trial: patients with a history of CVD or insulin therapy were not eligible for inclusion in this study. Indeed, there were relatively few patients who had low GSM lesions in the carotid wall. Although a previous study reported that carotid GSM values in the range of $30-40$ are considered 
to be an adequate cutoff to detect individuals at high risk for CVD $[39,40]$, the subjects who had low GSM lesions (GSM-CCA < 40) in their carotid walls at baseline represented only $10-20 \%$ of all subjects in this study. This may have led to the beneficial effects of alogliptin on the tissue characteristics of the carotid wall being underestimated. Finally, the subjects included in this study were Asian T2DM patients without the severe obesity that is often observed in nonAsian T2DM patients. It would thus be premature to generalize our findings to non-Asian populations.

\section{CONCLUSIONS}

A post hoc subanalysis suggests that the tissue characteristics of the carotid arterial wall were improved in both the alogliptin treatment group and the conventional treatment group during the 104-week treatment period and that there was no significant difference between the treatment groups. Prespecified studies with large sample size would be necessary to confirm our findings.

\section{ACKNOWLEDGEMENTS}

Funding. This research was supported by Grants-in-Aid for Scientific Research from the Japanese Ministry of Education, Science, Sports, Culture and Technology (KAKENHI 16K09747). The journal's article processing charges were also funded from the KAKENHI 16K09747.

Medical Writing Assistance. The authors gratefully acknowledge the assistance of $\mathrm{H}$. Yamada and D. Takayama (Soiken Holdings Inc, Tokyo Japan) in performing statistical analysis and R. Kondo (WILL Medical Communications) in proofreading the manuscript. All authors had full access to all of the data in this study and take complete responsibility for the integrity of the data and accuracy of the data analysis.

Authorship. All named authors meet the International Committee of Medical Journal
Editors (ICMJE) criteria for authorship for this manuscript, take responsibility for the integrity of the work as a whole, and have given final approval for the version to be published.

Disclosures. Yoko Irie has nothing to disclose. Naoto Katakami holds an endowed chair (Department of Metabolism and Atherosclerosis) established by funds from Kowa Pharmaceutical Co., has received research funds from MSD and lecture fees from Arkray Co. Ltd., Astellas Pharma Inc., Boehringer Ingelheim, Daiichi Sankyo Inc., Dainippon Sumitomo Pharma Co., Eli Lilly, Kowa Pharmaceutical Co., Kyowa Hakko Kirin Co. Ltd., Mitsubishi Tanabe Pharma Co., Novo Nordisk Pharma, Ono Pharmaceutical Co., Takeda Pharmaceutical Co., Sanofi-Aventis, and Shionogi \& Co. Tomoya Mita received research funds from MSD and Takeda Pharma K.K. and has received lecture fees from AstraZeneca K.K., Boehringer Ingelheim, Eli Lilly, Kowa Pharmaceutical Co., Mitsubishi Tanabe Pharma Co., MSD, Ono Pharmaceutical Co., and Takeda Pharmaceutical Co. Mitsuyoshi Takahara holds an endowed chair (Department of Diabetes Care Medicine) established by AstraZeneca K.K., Boehringer Ingelheim, Mitsubishi Tanabe Pharma Co., MSD, Novo Nordisk Pharma, Ono Pharmaceutical Co., Taisho Toyama Pharmaceutical Co. Taka-aki Matsuoka is an associate professor (Department of Metabolic Medicine) and received research funds from Daiichi Sankyo Inc., Ono Pharmaceutical Co, Eli Lilly, Novo Nordisk Pharma, Takeda Pharmaceutical Co. and lecture fees from MSD, Astellas Pharma Inc., Boehringer Ingelheim, Daiichi Sankyo Inc., Dainippon Sumitomo Pharma Co., Eli Lilly, Kowa Pharmaceutical Co., Kyowa Hakko Kirin Co. Ltd., Mitsubishi Tanabe Pharma Co., Novo Nordisk Pharma, Ono Pharmaceutical Co., Kissei Pharmaceutical Co., Taisho Toyama Pharmaceutical Co., Ltd., Sanwa Kagaku Kenkyusho Co., Sanofi Co. Masahiko Gosho received lecture and/or consultant fees from Daiichi Sankyo Company, Ltd., Novartis, Taiho Pharma, and Ferring Pharma, received travel fees from Takeda Pharmaceutical Co., and received manuscript fees from Kowa Co. Ltd. Hirotaka Watada received lecture fees from Novo 
Nordisk, Inc., Eli Lilly and Company, Sanofi, Dainippon Sumitomo Pharma Co., Fujifilm, Bayer Health Care, Kissei Pharmaceutical Company, Mochida Pharmaceutical Company, MSD, Takeda Pharmaceutical Company, Boehringer Ingelheim Pharmaceuticals Inc., Daiichi-Sankyo, Ono Pharmaceutical Co. Ltd., Novartis Pharmaceuticals Corporation, Mitsubishi Tanabe Pharma Corporation, AstraZeneca LP, Kyowa Hakko Kirin Company Ltd., Sanwa Kagaku Kenkyusho Co. Ltd., Kowa Company Ltd., Astellas Pharma Inc.; advisory fees from Novo Nordisk, Inc., Mochida Pharma Company, AstraZeneca LP, Kowa Company, Astellas Pharma Inc., Sanofi, Boehringer Ingelheim Pharmaceuticals Inc., MSD, Mitsubishi Tanabe Pharma Corporation, Novartis Pharmaceuticals Corporation, Dainippon Sumitomo Pharma Co., Takeda Pharmaceutical Company, Ono Pharmaceutical Co., Pfizer Inc., and Kowa Company; and research funds from Boehringer Ingelheim, Pfizer, Mochida Pharmaceutical Co., Sanofi-Aventis, Novo Nordisk Pharma, Novartis Pharmaceuticals, Sanwakagaku Kenkyusho, Terumo Corp. Eli Lilly, Mitsubishi Tanabe Pharma, Daiichi Sankyo Inc., Takeda Pharmaceutical Co., MSD, Shionogi, Pharma, Dainippon Sumitomo Pharma, Kissei Pharma, and Astrazeneca K.K. Iichiro Shimomura received lecture fees from Astellas Pharma Inc., AstraZeneca K.K., MSD K.K., Ono Pharmaceutical Co., Kyowa Hakko Kirin Co., Kowa Pharmaceutical Co., Sanofi K.K., Sanwa Kagaku Kenkyusho Co., Daiichi Sankyo Co., Takeda Pharma K.K., Mitsubishi Tanabe Pharma Co., Teijin Pharma, Eli Lilly Japan K.K., Nippon Boehringer Ingelheim Co., Novartis Pharma K.K., Novo Nordisk Pharma, Bayer Yakuhin, Pfizer Japan Inc., Bristol-Myers K.K., Mochida Pharmaceutical Co., Shionogi \& Co., and Taisho Toyama Pharmaceutical Co., and research funds from Astellas Pharma Inc., AstraZeneca K.K., Eisai Co., MSD K.K, Otsuka Pharmaceutical Co., Ono Pharmaceutical Co., Kaken Pharmaceutical Co., Kissei Pharmaceutical Co., Kyowa Hakko Kirin Co., Sanofi K.K., Shionogi \& Co., Daiichi Sankyo Co., Dainippon Sumitomo Pharma Co., Takeda Pharma K.K., Mitsubishi Tanabe Pharma Co., Teijin Pharma, Nippon Boehringer Ingelheim Co., Novartis Pharma K.K., Novo Nordisk
Pharma, Pfizer Japan Inc., Bristol-Myers K.K., Mochida Pharmaceutical Co., Eli Lilly Japan K.K, Kowa Co., Ltd., Kowa Pharmaceutical Co., and Taisho Toyama Pharmaceutical Co.

Compliance with Ethics Guidelines. This article is based on previously conducted studies and does not involve any new studies of human or animal subjects performed by any of the authors.

Open Access. This article is distributed under the terms of the Creative Commons Attribution-NonCommercial 4.0 International License (http://creativecommons.org/licenses/ by-nc/4.0/), which permits any noncommercial use, distribution, and reproduction in any medium, provided you give appropriate credit to the original author(s) and the source, provide a link to the Creative Commons license, and indicate if changes were made.

\section{REFERENCES}

1. Fuster V, Badimon L, Badimon JJ, Chesebro JH. The pathogenesis of coronary artery disease and the acute coronary syndromes. $\mathrm{N}$ Engl J Med. 1992;326:242-50.

2. Lee RT, Grodzinsky AJ, Frank EH, Kamm RD, Schoen FJ. Structure-dependent dynamic mechanical behavior of fibrous caps from human atherosclerotic plaques. Circulation. 1991;83:1764-70.

3. Davies MJ, Richardson PD, Woolf N, Katz DR, Mann J. Risk of thrombosis in human atherosclerotic plaques: role of extracellular lipid, macrophage, and smooth muscle cell content. $\mathrm{Br}$ Heart J. 1993;69:377-81.

4. Falk E. Pathogenesis of atherosclerosis. J Am Coll Cardiol. 2006;47:C7-12.

5. Creager MA, Lüscher TF, Cosentino F, Beckman JA. Diabetes and vascular disease: pathophysiology, clinical consequences, and medical therapy: part I. Circulation. 2003;108:1527-32.

6. Grønholdt ML, Wiebe BM, Laursen H, Nielsen TG, Schroeder TV, Sillesen H. Lipid-rich carotid artery plaques appear echolucent on ultrasound B-mode images and may be associated with intraplaque 
haemorrhage. Eur J Vasc Endovasc Surg. 1997;14:439-45.

7. Nicolaides AN, Kakkos SK, Kyriacou E, et al. Asymptomatic internal carotid artery stenosis and cerebrovascular risk stratification. J Vasc Surg. 2010;52:1486-96.

8. Irie $\mathrm{Y}$, Katakami N, Kaneto H, et al. The utility of ultrasonic tissue characterization of carotid plaque in the prediction of cardiovascular events in diabetic patients. Atherosclerosis. 2013;230:399-405.

9. Grønholdt ML, Nordestgaard BG, Schroeder TV, Vorstrup S, Sillesen H. Ultrasonic echolucent carotid plaques predict future strokes. Circulation. 2001;104:68-73.

10. Fadini GP, Avogaro A. Cardiovascular effects of DPP-4 inhibition: beyond GLP-1. Vasc Pharmacol. 2011;55:10-6.

11. Barrera JG, Sandoval DA, D'Alessio DA, Seeley RJ. GLP-1 and energy balance: an integrated model of short-term and long-term control. Nat Rev Endocrinol. 2011;7:507-16.

12. Lovshin JA, Drucker DJ. Incretin-based therapies for type 2 diabetes mellitus. Nat Rev Endocrinol. 2009;5:262-9.

13. Ban K, Noyan-Ashraf MH, Hoefer J, Bolz SS, Drucker DJ, Husain M. Cardioprotective and vasodilatory actions of glucagon-like peptide 1 receptor are mediated through both glucagon-like peptide 1 receptor dependent and independent pathways. Circulation. 2008;117:2340-50.

14. Liu H, Dear AE, Knudsen LB, Simpson RW. A longacting glucagon-like peptide-1 analogue attenuates induction of plasminogen activator inhibitor type-1 and vascular adhesion molecules. J Endocrinol. 2009;201:59-66.

15. Hattori Y, Jojima T, Tomizawa A, et al. A glucagonlike peptide-1 (GLP-1) analogue, liraglutide, upregulates nitric oxide production and exerts anti-inflammatory action in endothelial cells. Diabetologia. 2010;53:2256-63.

16. Arakawa M, Mita T, Azuma K, et al. Inhibition of monocyte adhesion to endothelial cells and attenuation of atherosclerotic lesion by a glucagon-like peptide-1 receptor agonist, exendin-4. Diabetes. 2010;59:1030-7.

17. Gaspari T, Liu H, Welungoda I, et al. A GLP-1 receptor agonist liraglutide inhibits endothelial cell dysfunction and vascular adhesion molecule expression in an ApoE-/- mouse model. Diabetes Vasc Dis Res. 2011;8:117-24.
18. Nagashima M, Watanabe T, Terasaki $M$, et al. Native incretins prevent the development of atherosclerotic lesions in apolipoprotein $\mathrm{E}$ knockout mice. Diabetologia. 2011;54:2649-59.

19. Goto H, Nomiyama $T$, Mita $T$, et al. Exendin-4, a glucagonlike peptide-1 receptor agonist, reduces intimal thickening after vascular injury. Biochem Biophys Res Commun. 2011;405:79-84.

20. Tanaka M, Matsuo Y, Yamakage H, et al. Differential effects of GLP-1 receptor agonist on foam cell formation in monocytes between non-obese and obese subjects. Metabolism. 2016;65:1-11.

21. Terasaki M, Nagashima $M$, Nohtomi $K$, et al. Preventive effect of dipeptidyl peptidase- 4 inhibitor on atherosclerosis is mainly attributable to incretin's actions in nondiabetic and diabetic apolipoprotein E-null mice. PLoS One. 2013;8:e70933.

22. Terasaki $Y$, Nomiyama $T$, Kawanami $T$, et al. Dipeptidyl peptidase-4 inhibitor linagliptin attenuates neointima formation after vascular injury. Cardiovasc Diabetol. 2014;13:154.

23. Nader MA. Sitagliptin ameliorates lipid profile changes and endothelium dysfunction induced by atherogenic diet in rabbits. Naunyn Schmiedebergs Arch Pharmacol. 2014;387:433-44.

24. Hirano T, Mori Y. Anti-atherogenic and anti-inflammatory properties of glucagon-like peptide-1, glucose-dependent insulinotropic polypepide, and dipeptidyl peptidase- 4 inhibitors in experimental animals. J Diabetes Investig. 2016;7:80-6.

25. Balestrieri ML, Rizzo MR, Barbieri M, et al. Sirtuin 6 expression and inflammatory activity in diabetic atherosclerotic plaques: effects of incretin treatment. Diabetes. 2015;64:1395-406.

26. Vittone F, Liberman A, Vasic D, et al. Sitagliptin reduces plaque macrophage content and stabilises arteriosclerotic lesions in Apoe (-/-) mice. Diabetologia. 2012;55:2267-75.

27. Satoh-Asahara N, Sasaki Y, Wada H, et al. A dipeptidyl peptidase- 4 inhibitor, sitagliptin, exerts antiinflammatory effects in type 2 diabetic patients. Metabolism. 2013;62:347-51.

28. Tremblay AJ, Lamarche B, Deacon CF, Weisnagel SJ, Couture P. Effects of sitagliptin therapy on markers of low-grade inflammation and cell adhesion molecules in patients with type 2 diabetes. Metabolism. 2014;63:1141-8.

29. Mita T, Katakami N, Yoshii H, et al. Alogliptin, a dipeptidyl peptidase 4 inhibitor, prevents the progression of carotid atherosclerosis in patients with 
type 2 diabetes: the study of preventive effects of alogliptin on diabetic atherosclerosis (SPEAD-A). Diabetes Care. 2016;39:139-48.

30. Terminology and Diagnostic Criteria Committee, Japan Society of Ultrasonics in Medicine, Subcommittee for Preparing Guidelines for Ultrasound Diagnosis of Carotid Artery. Standard method for ultrasound evaluation of carotid artery lesions. Jpn J Med Ultrason. 2009;36:501-18.

31. Yanase T, Nasu S, Mukuta Y, et al. Evaluation of a new carotid intima-media thickness measurement by B-mode ultrasonography using an innovative measurement software, intimascope. Am J Hypertens. 2006;19:1206-12.

32. Sabetai MM, Tegos TJ, Nicolaides AN, Dhanjil S, Pare GJ, Stevens JM. Reproducibility of computerquantified carotid plaque echogenicity: can we overcome the subjectivity? Stroke. 2000;31:2189-96.

33. Libby P, Aikawa M. Stabilization of atherosclerotic plaques: new mechanisms and clinical targets. Nat Med. 2002;8:1257-62.

34. Irie $\mathrm{Y}$, Katakami N, Kaneto $\mathrm{H}$, et al. The risk factors associated with ultrasonic tissue characterization of carotid plaque in type 2 diabetic patients. J Diabetes Complicat. 2014;28:523-7.
35. Yamasaki Y, Kodama M, Nishizawa H, et al. Carotid intima-media thickness in Japanese type 2 diabetic subjects: predictors of progression and relationship with incident coronary heart disease. Diabetes Care. 2000;9:1310-5.

36. Katakami N, Sakamoto K, Kaneto H, et al. Lipidlowering with atorvastatin improves tissue characteristics of carotid plaque. Atherosclerosis. 2005;183:369-71.

37. Crisby M, Nordin-Fredriksson G, Shah PK, Yano J, Zhu J, Nilsson J. Pravastatin treatment increases collagen content and decreases lipid content, inflammation, metalloproteinases, and cell death in human carotid plaques: implications for plaque stabilization. Circulation. 2001;103:926-33.

38. Makris GC, Lavida A, Nicolaides AN, Geroulakos G. The effect of statins on carotid plaque morphology: an LDL-associated action or one more pleiotropic effect of statins? Atherosclerosis. 2010;213:8-20.

39. Falkowski A, Kaczmarczyk M, Cieszanowski A, Goracy I, Poncyliusz W, Wilk G. Computer-assisted characterisation of a carotid plaque. Med Sci Monit. 2004;10:67-70.

40. Nicolaides AN, Kakkos SK, Kyriacou E, et al. Asymptomatic internal carotid artery stenosis and cerebrovascular risk stratification. J Vasc Surg. 2010;52:1486-96. 